\title{
Spectrum of imaging findings in pulmonary infections. Part 1: Bacterial and viral
}

\author{
Mandeep Garg ${ }^{A, B, D, E, F}$, Nidhi Prabhakar ${ }^{A, B, E, F}$, Ajay Gulati ${ }^{D, E, F}$, Ritesh Agarwal ${ }^{B, E, F}$, Sahajal Dhooria ${ }^{B, E, F}$ \\ Postgraduate Institute of Medical Education and Research, India
}

\section{Abstract}

\begin{abstract}
Chest radiography is generally the first imaging modality used for the evaluation of pneumonia. It can establish the presence of pneumonia, determine its extent and location, and assess the response to treatment. Computed tomography is not used for the initial evaluation of pneumonia, but it may be used when the response to treatment is unusually slow. It helps to identify complications, detect any underlying chronic pulmonary disease, and also to characterise complex pneumonias. Although not diagnostic, certain imaging findings may suggest a particular microbial cause over others. Knowledge of whether pneumonia is community-acquired or nosocomial, as well as the age and immune status of the patient, can help us in narrowing the differential diagnoses. The purpose of this article is to briefly review the various pulmonary imaging manifestations of pathogenic organisms. This knowledge along with clinical history and laboratory investigations of the patient may help in guiding the treatment of pneumonia.
\end{abstract}

Key words: fungal, bacterial, pneumonia, viral, mycobacterial, parasitic.

\section{Introduction}

Pneumonia is defined as an infection of the lower respiratory tract, which involves the lung parenchyma. Pneumonitis and pneumonia are terms that refer to a similar pathology and can be used interchangeably. However, the term 'pneumonia' usually refers to infection by a pathogenic organism, which results in consolidation of the lung, whereas the term pneumonitis is generally used in the context of non-infective inflammation that primarily involves the alveolar wall [1].

Chest radiographs are the most widely used imaging modality followed by cross-sectional imaging methods like computed tomography (CT). A chest radiograph can establish the presence of pneumonia, determine its extent and location, and assess the response to treatment. It can also diagnose complications like pleural effusion, pneumothorax, and abscess formation. CT may detect abnormalities that are not appreciable on chest radiograph. It is not used for the initial evaluation of pneumonia but may be used when the response to treatment is unusually slow, to look for complications, to detect underlying disease within the lung, and also to characterise any complex pneumonias. The main role of chest ultrasonography in pneumonia is to assess pleural effusion or empyema and serve as a guide for aspiration/drainage. Recently, data has been published regarding the utility of magnetic resonance imaging (MRI) as a radiation-free technique for diagnosing pulmonary infections $[2,3]$.

Pneumonia was classically divided according to its morphological pattern on imaging into the following: lobar pneumonia, bronchopneumonia, and interstitial pneumonia [4]. Lobar consolidation is characterised by confluent areas of consolidation, which are usually confined to one segment or lobe. In bronchopneumonia, there are centrilobular nodules that coalesce to cause consolidation, and involve one or more lobes. Interstitial pneumonia is caused by the involvement of bronchial or bronchiolar wall and pulmonary interstitium and leads to septal thickening, nodules, and areas of ground glass opacities (GGO) on imaging [5]. This classification is no longer commonly used because, with new organisms and widespread use of

\section{Correspondence address:}

Dr. Mandeep Garg, MD, FRCR, Prof., Department of Radiodiagnosis and Imaging, PGIMER, Chandigarh, India, e-mail: gargmandeep@hotmail.com

Authors' contribution:

A Study design · B Data collection · C Statistical analysis · D Data interpretation · E Manuscript preparation · F Literature search · G Funds collection 

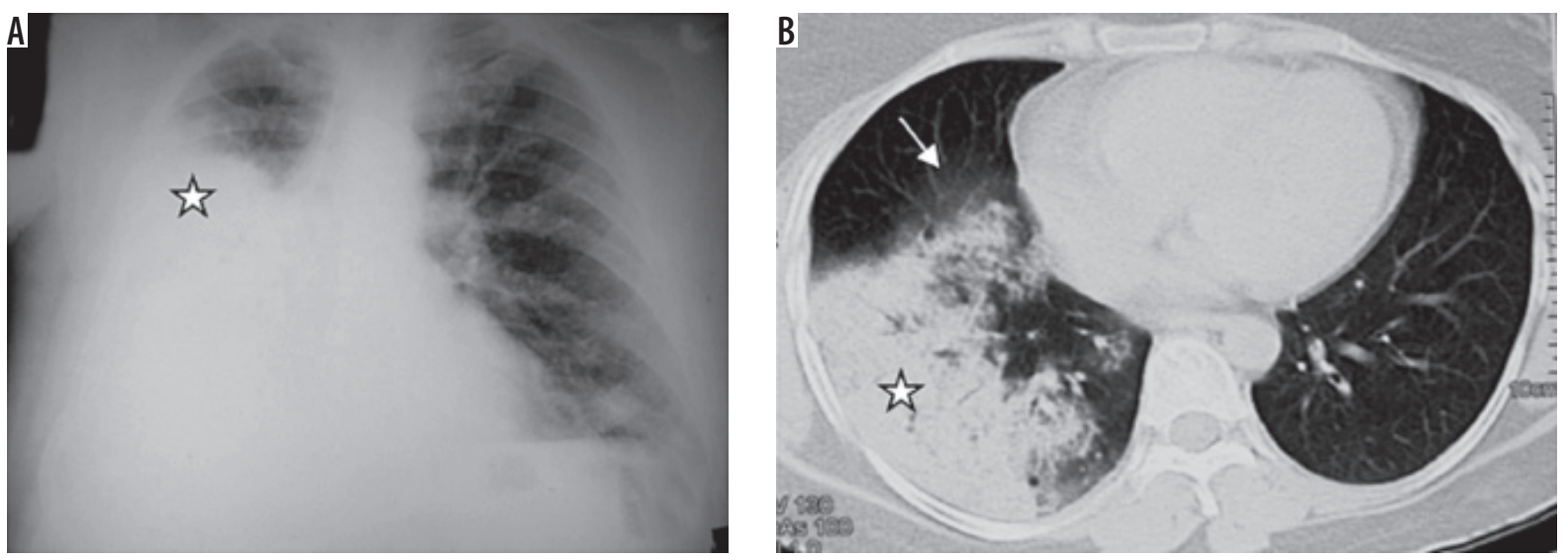

Figure 1. Streptococcal pneumonia in a 35-year-old patient. A) Chest radiograph showing right middle and lower zone consolidation (asterisk) and associated pleural effusion. B) Chest computed tomography shows the presence of consolidation in the right lower lobe (asterisk) with ground glass opacities in the periphery (arrow)

antibiotics, morphological patterns nowadays are mostly overlapping. In the current era, pneumonias are preferably classified as community-acquired, nosocomial, or aspiration pneumonia, depending on their origin.

\section{Bacterial pneumonia}

The most common causes of community-acquired pneumonia (CAP) are Streptococcus pneumonia, Haemophilus influenzae, Mycoplasma pneumoniae, Chlamydia pneumoniae, and Legionella pneumophila. Aerobic Gram-negative bacilli, particularly Pseudomonas aeruginosa and Enterobacteriae and a few Gram-positive cocci such as Staphylococcus aureus are more commonly responsible for hospital-acquired pneumonia (HAP). The cause of aspiration pneumonias are micro-organisms that colonise the oropharynx; these include Gram-positive cocci, Gram-negative rods, and anaerobic bacteria [6,7].

Streptococcus pneumonia is the most common aetiological agent producing CAP. Lobar pneumonia is the most common imaging appearance. It predominantly involves lower lobes (Figure 1). The lung volume is not altered, and cavitation is rare. Patchy areas of consolidation that may be multilobar or bilateral have been seen in patients, following antibiotic therapy. A small amount of reactive pleural effusion is usually seen [8]. Centrilobular nodules, bronchial wall thickening, and bronchiectasis are seen in patients who are concurrently infected with other organisms [9].

Staphylococcus aureus can cause hospital-acquired or ventilator-associated pneumonia, fulminating secondary pneumonia after a viral infection, and pneumonia associated with pneumatoceles in infants [10]. Radiographic findings include mostly the bronchopneumonia pattern. CT will show centrilobular nodules and bronchial wall thickening. Lung parenchymal abnormalities in the form of peripheral areas of consolidation are more frequently seen in methicillin-resistant staphylococcal bacteria [11]. Cavitation may appear in areas of consolidation, forming lung abscesses; empyema may also be encountered.
Pneumatoceles (thin-walled, air-filled cystic spaces) can appear during the first week and disappear spontaneously within weeks or months [10]. Pneumatoceles can lead to secondary pneumothorax (Figure 2).

Mycoplasma pneumoniae most commonly affects young people. The chest radiograph may show reticulonodular opacities or patchy areas of consolidations [12]. On CT, centrilobular nodules, GGO, and lobular areas of consolidation with bronchial wall thickening are common findings (Figure 3) $[13,14]$. Extensive GGO or consolidation can progress to acute respiratory distress syndrome (ARDS). Imaging appearance of chlamydia is almost similar to mycoplasma. Radiograph shows a bronchopneumonia pattern. The interstitial pattern is less common. On CT, consolidation with bronchial wall thickening is the predominant finding [15].

Legionnaires' disease is caused by Legionella pneumophila. This infection usually appears in outbreaks and is spread by inhaling droplets of contaminated water, for example through air conditioners and humidifiers [16]. Chest radiograph shows a rapidly progressing consolidation, which is usually segmental and peripheral initially and becomes bilateral in 50\% of cases (Figure 4). CT shows multilobar/multisegmental air space disease interspersed with GGO. Imaging findings lag behind clinical improvement [16]. There is a complete resolution in most cases after treatment. Early identification of this pneumonia is important because a delay in diagnosis can be fatal.

Klebsiella pneumonia (Friedlander's pneumonia) usually develops in elderly patients with chronic pulmonary disease or in alcoholics. Radiological findings include homogeneous parenchymal consolidation containing air bronchograms [17]. The right upper lobe is most commonly involved. Infection usually induces large inflammatory exudate, leading to an increased volume of the affected lobe. This increased volume causes characteristic bulging of an adjacent interlobar fissure (Figure 5). The complications consist of chronic pneumonia, lung abscess, and pulmonary gangrene. Centrilobular nodules, 

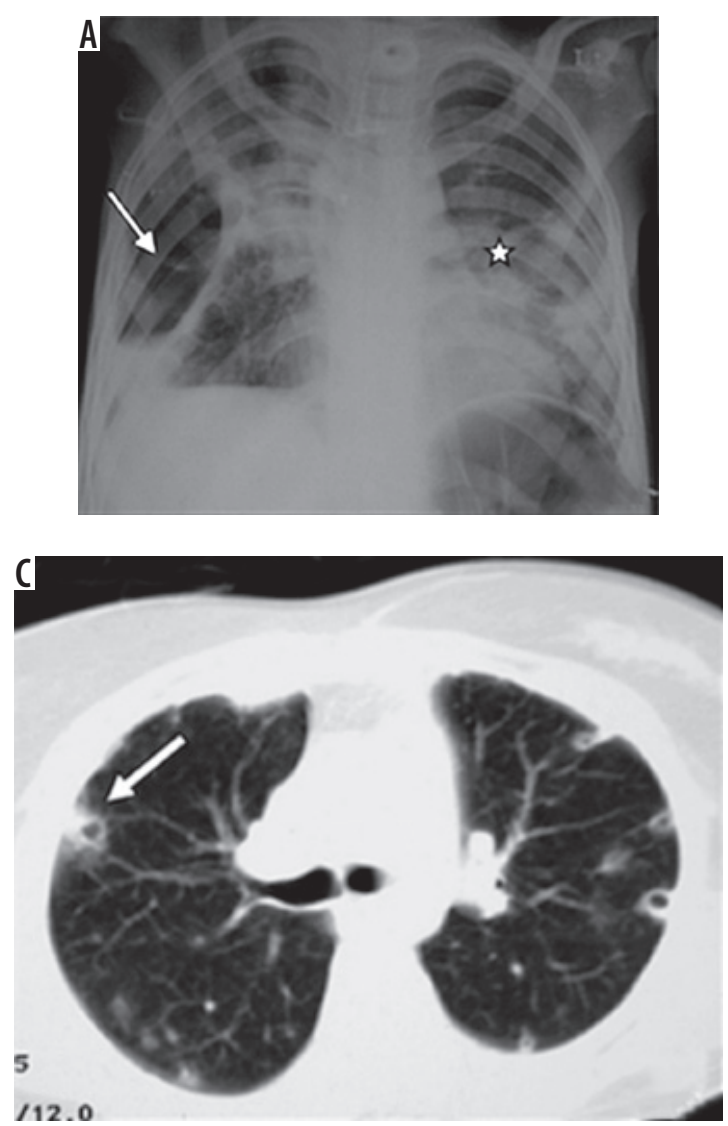
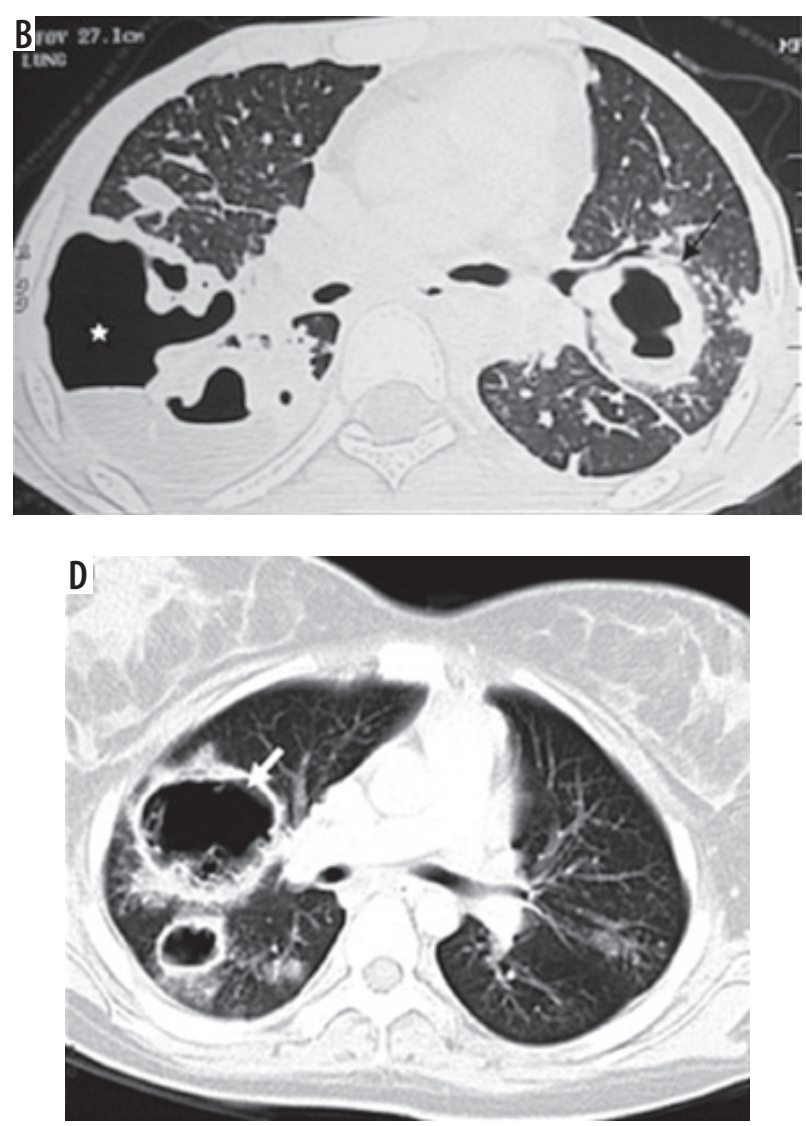

Figure 2. Staphylococcal pneumonia. A) Chest radiograph shows hydropneumothorax (white arrow) on the right side and consolidation with cavitation in the left mid and lower zones (asterisk). B) Chest computed tomography (CT) image shows presence of thick-walled cavity with rupture into right pleural cavity (asterisk) and thick-walled cavity, with adjacent centrilobular nodules, in left upper lobe (black arrow). C) Chest CT in a different patient shows cavitating nodules (white arrow). D) Chest (T in another patient showing pneumatoceles in right upper lobe, following staphylococcal pneumonia (white arrow)

bronchial wall thickening, and bronchiectasis are seen in patients with other concurrent infections [18].

Gram-negative aerobic pneumonia other than Klebsiella includes Haemophilus, Pseudomonas, Escherichia coli, Proteus, etc. They usually cause nosocomial and CAP. The predisposed population includes those with underlying local/systemic disease and debilitated old-age people. Imaging findings include a bronchopneumonia pattern with centrilobular nodules, bronchial wall thickening, GGO, and patchy consolidation, which later leads to lobar distribution. Pleural effusion and cavitation are more common (Figure 6) [19].

Among the anaerobes, Bacteroides, Fusobacterium, and Actinomyces are the most common pathogens. They are the predominant organisms causing aspiration pneumonia. Most infections are polymicrobial [8]. Alcoholism, poor oral hygiene, and unconsciousness are considered to be risk factors. Most commonly affected are the posterior segment of the upper lobes and the superior segments of the lower lobes in the recumbent patient, and basal segments of the lower lobes in erect patients. The right lung is usually involved more than the left lung. Radiographically a bronchopneumonia pattern is seen ranging from segmental areas of patchy, bilateral opacities to extensive, confluent disease. These patients may show cavitation or abscess formation (Figure 7) [20].

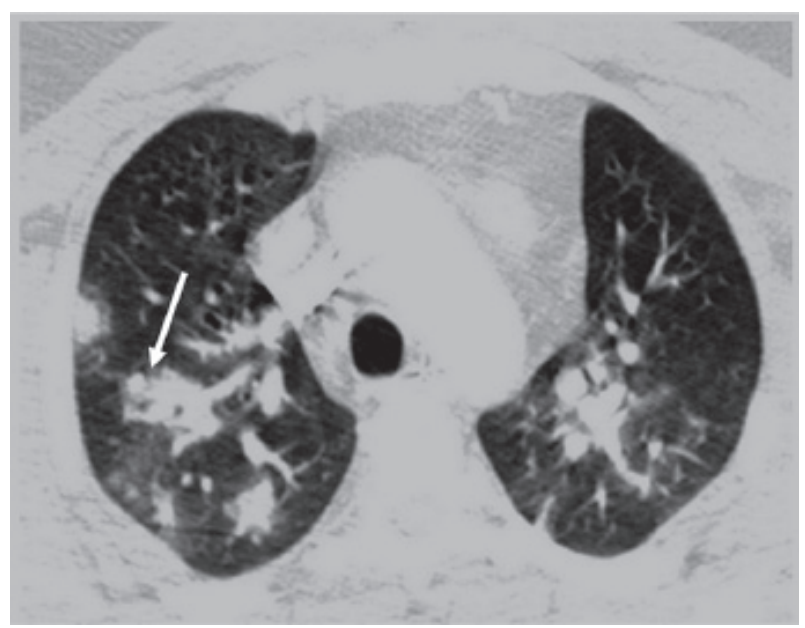

Figure 3. Mycoplasma pneumonia in an adult patient. Chest computed tomography demonstrates peribronchial areas of consolidation with centrilobular nodules and adjacent ground glass opacities (long arrow)

Nocardiosis predominantly leads to infections in immunocompromised hosts. The most common pathogen is Nocardia aesteroides. Radiographic findings include multifocal areas of consolidation. Areas of low attenuation may be present within consolidated lung, probably reflecting abscess formation. Cavitation occurs in up to $30 \%$ of cases. Other findings include centrilobular nodules, periphe- 

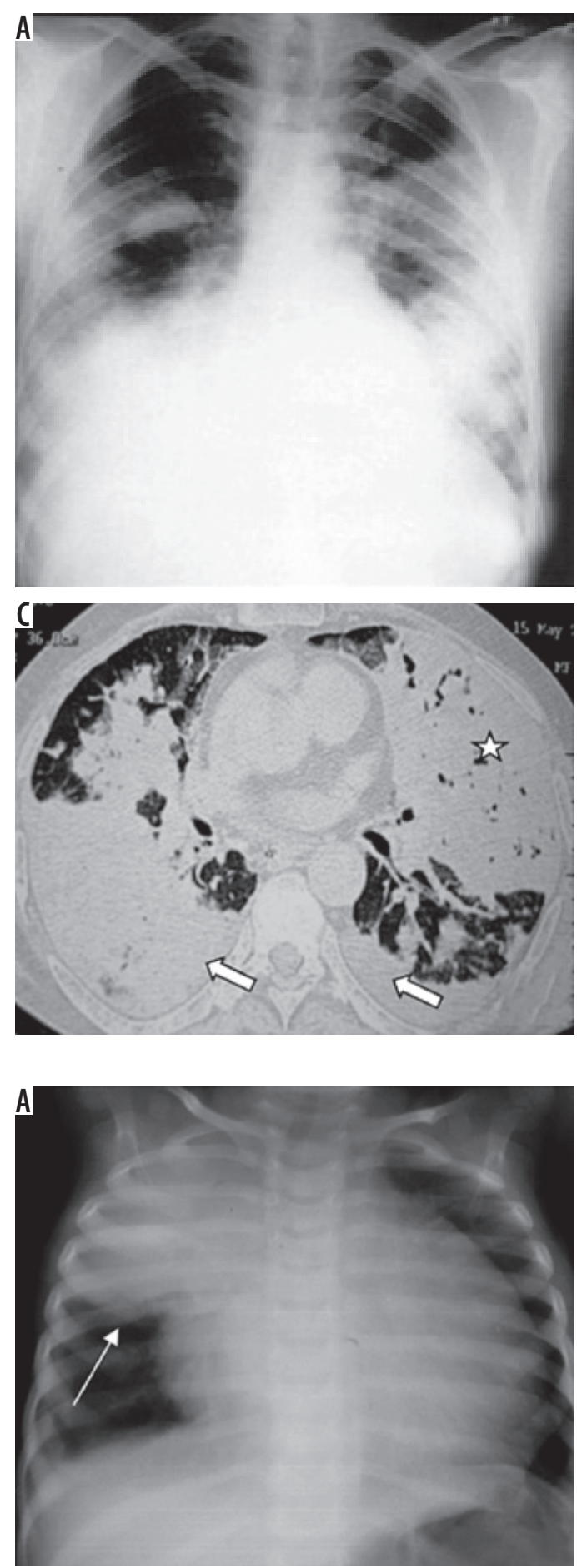

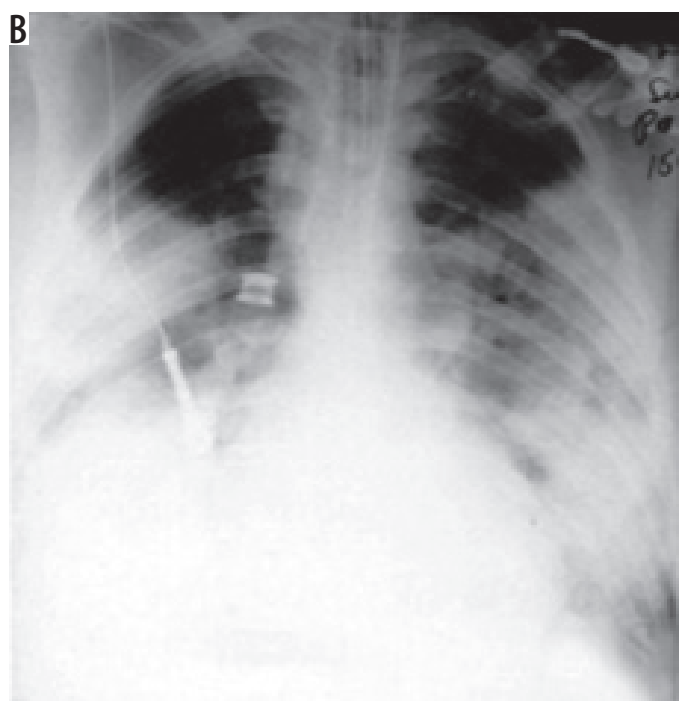

Figure 4. Legionella pneumonia in an adult male patient. A, B) Sequential chest radiographs show the presence of rapidly progressing bilateral lobar consolidation. C) Chest computed tomography shows lobar consolidation (asterisk) with bilateral pleural effusion (block arrows)

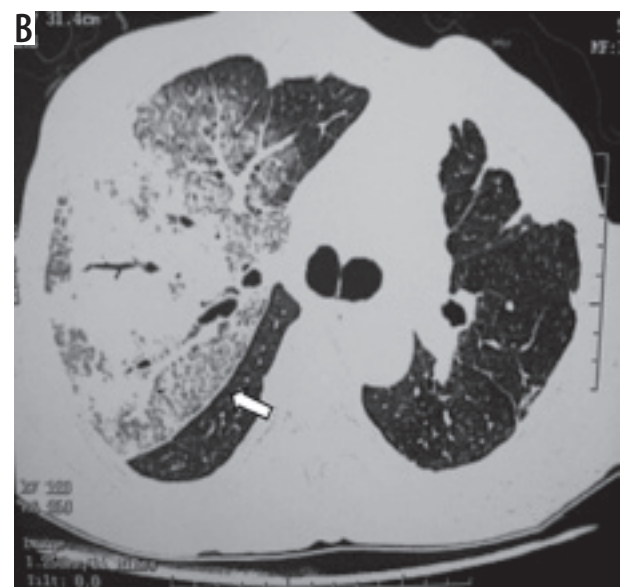

Figure 5. Klebsiella pneumonia in two different patients. A) Chest radiograph in a paediatric patient typically shows lobar consolidation with bulging fissure (long arrow). B) Chest computed tomography in another patient with Klebsiella pneumonia demonstrates lobar consolidation with bulging fissure and air bronchogram (short block arrow)

ral GGO, and pleural/chest wall involvement (Figure 8). Chronic cases can show pleural thickening, bronchiectasis, and collection in the chest wall [21].

\section{Viral pneumonia}

Viral respiratory infections are common in children. In infants and young children, the most common viral infections are respiratory syncytial virus (RSV), parainfluenza, adenovirus, and influenza virus [22]. Influenza type A and $\mathrm{B}$ and adenovirus are more commonly seen in adults. In immunocompromised patients, viral respiratory infections are usually secondary to cytomegalovirus (CMV) and other herpes viruses [23]. Clinical symptoms are nonspecific, and the clinical course of infection depends on age, the infectious agent, and the immune status of the patient [24]. The nasopharynx and upper respiratory tract are usually involved during the initial period of illness. 


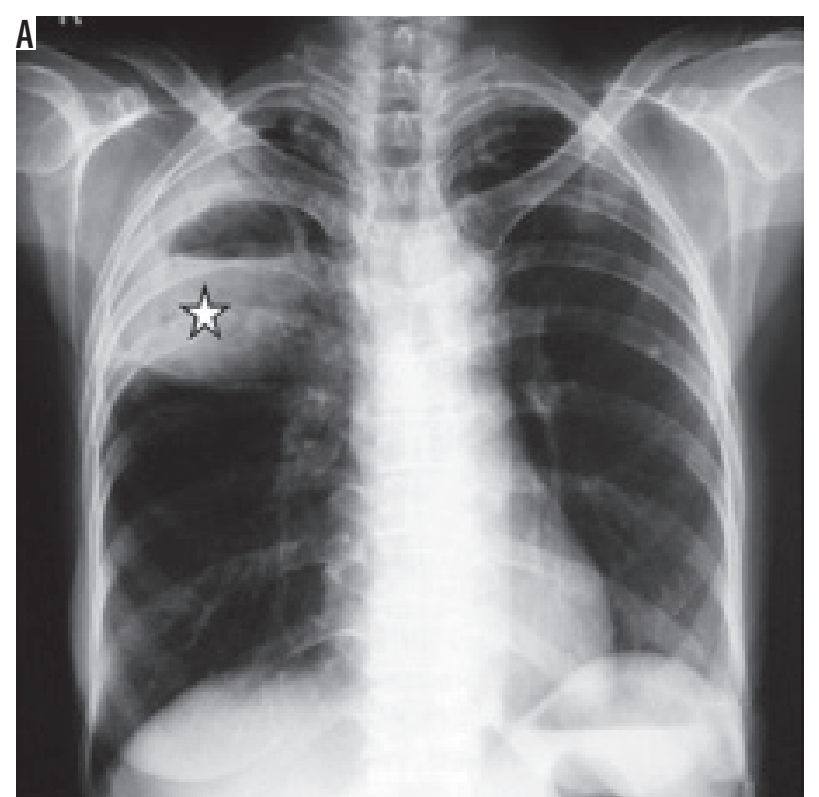

The disease subsequently migrates to small airways and alveoli [20], leading to tracheobronchitis, bronchiolitis, and pneumonia. Pneumonia is initially interstitial and later involves lung adjacent to terminal bronchioles. Viral infections predispose to superimposed bacterial infection.

Influenza virus infections can occur in the form of epidemics or pandemics, or sporadically. Severe outbreaks of pneumonia have been most commonly associated with influenza subtypes A and B. Influenza infection is more common in infants. It is also one of the important causes of pulmonary infection in immunocompromised patients. It may lead to rhinitis, pharyngitis, tracheobronchitis, and bronchiolitis. Pneumonia may be due to the virus itself or due to superimposed bacterial infection. Chest radiograph shows segmental consolidation, with basal predominance, which may become rapidly confluent to give an ARDSlike picture. CT shows diffuse bilateral GGO, consolidation, centrilobular nodules, and bronchial wall thickening (Figure 9). No difference is seen in the radiological findings of pneumonias caused by influenza $\mathrm{A}$ or $\mathrm{B}$ or various subtypes of A [25].

Respiratory syncytial virus is the most common cause of viral lower respiratory tract infection in children, leading to bronchiolitis and bronchopneumonia [26]. In adults, it may cause fever, but it rarely causes complications unless the patient is immunocompromised. The chest radiograph may show peribronchial infiltrates with associated overinflation. Sometimes lobar collapse/consolidation or enlarged hilar lymph nodes are seen. The most characteristic findings on CT in the early phase of the disease are centrilobular nodules and tree-in-bud appearance. Subsequent scans are less characteristic, with multiple CT variations, GGO, and nodules being the most common findings (Figure 10) [27]. Adenovirus is also known as the common cold virus, which may sometimes lead to pneumonia. It leads to

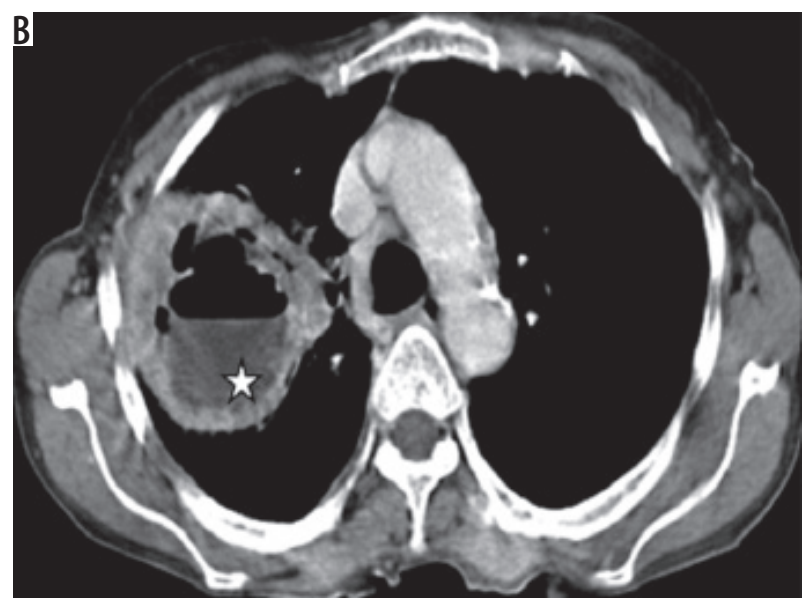

Figure 6. Escherichia coli lung abscess in an adult patient. A) Chest radiograph shows presence of cavity with air fluid level (white asterisk) in right upper lung zone. B) Chest computed tomography of the same patient shows presence of thick walled cavitary lesion with air-fluid level in right upper lobe (white asterisk)
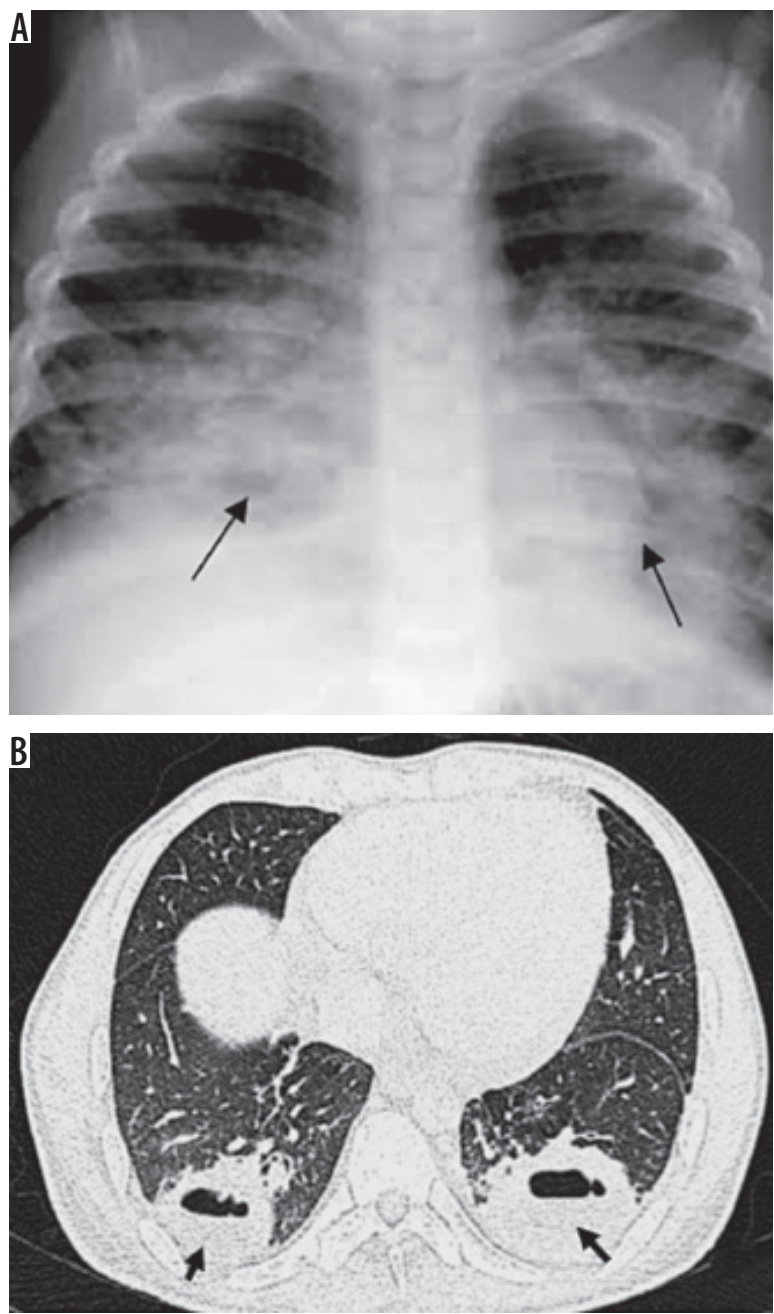

Figure 7. Aspiration pneumonia in two different patients. A) Chest radiograph shows presence of consolidation with breakdown and air fluid level in right lower lung zone and consolidation in left lower lung zone (long black arrows). B) Chest computed tomography image shows presence of thick-walled cavity with air-fluid level in bilateral lower lobes (short block arrows) posteriorly, consistent with aspiration 

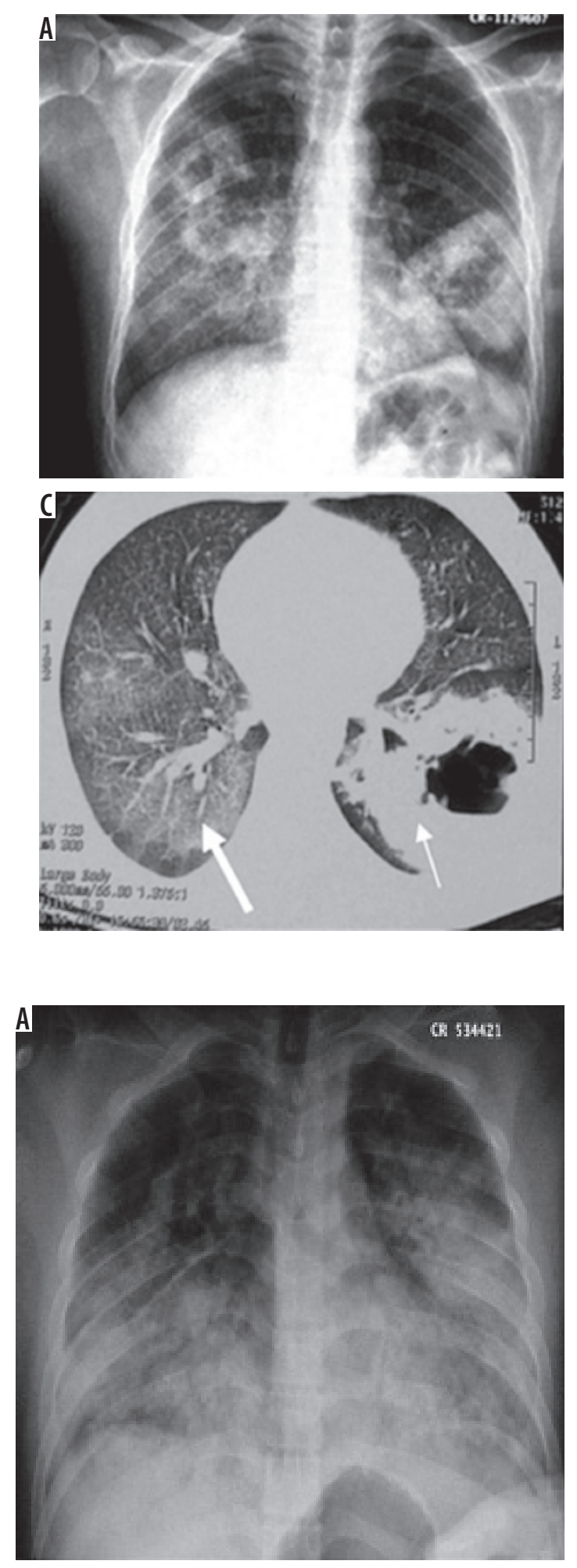

$5-10 \%$ of acute respiratory infections in infants and less than $1 \%$ in adults [28]. The chest radiograph may show scattered patchy/confluent opacities and hilar lymphadenopathy. CT may show bilateral consolidation, areas of GGO, nodules, and pleural effusion. CT findings may resemble bacterial pneumonia [29]. Infection in children before two years of age may lead to bronchiectasis, obliterative bronchiolitis, and Swyer-James/McLeod's syndrome [30]. CT in Swyer James syndrome may either show uni-

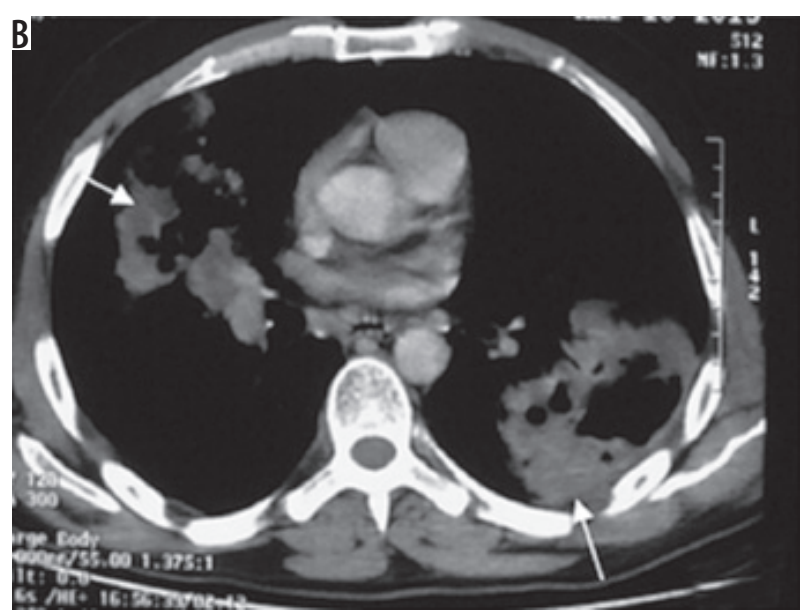

Figure 8. Post renal transplant patient with persistent fever and cough. Fine needle aspiration from the cavity showed Nocardia asteroids. A) Chest radiograph shows presence of multiple patches of consolidations with areas of cavitation in bilateral lungs. B, C) Chest computed tomography shows presence of patchy areas of consolidation with breakdown in left lower lobe (long white arrow) and right middle lobe (short white arrow). Diffuse areas of ground glass opacities are also seen in both lungs (block white arrow)

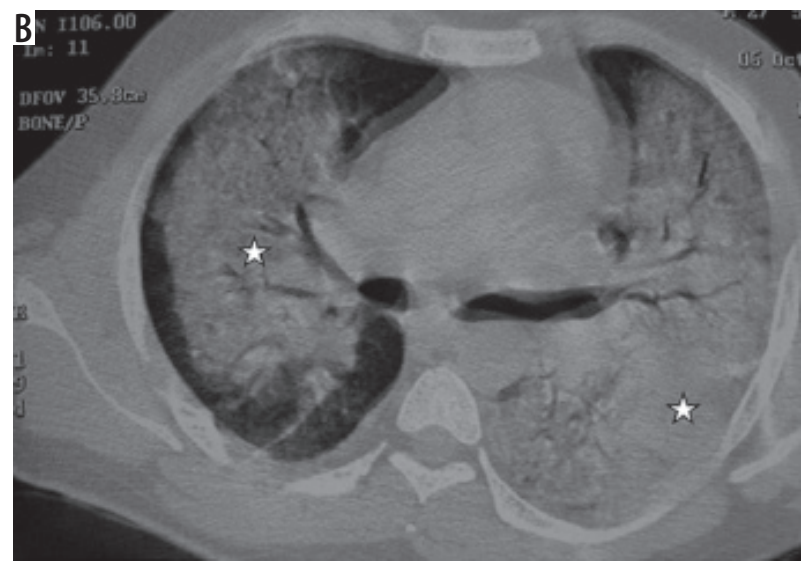

Figure 9. Patient with H1N1 viral pneumonia. A) Chest radiograph shows presence of bilateral consolidation with perihilar predominance. B) Chest computed tomography shows presence of bilateral perihilar consolidation (asterisk) with air bronchograms

lateral hyperlucency with hyperinflated lung, the collapse of the affected lung, unilateral hyperlucency of a small/ normal lung, bilateral hyperlucency, or a mixed pattern of hyperlucency / collapse with peribronchial thickening (Figure 11) [31].

Cytomegalovirus infection is seen in acquired immune deficiency syndrome (AIDS) patients (with CD4 cells $<100$ cells $/ \mathrm{mm}^{3}$ ) [26] and patients with haematopoietic stem cell and solid organ transplants. A third of patients 

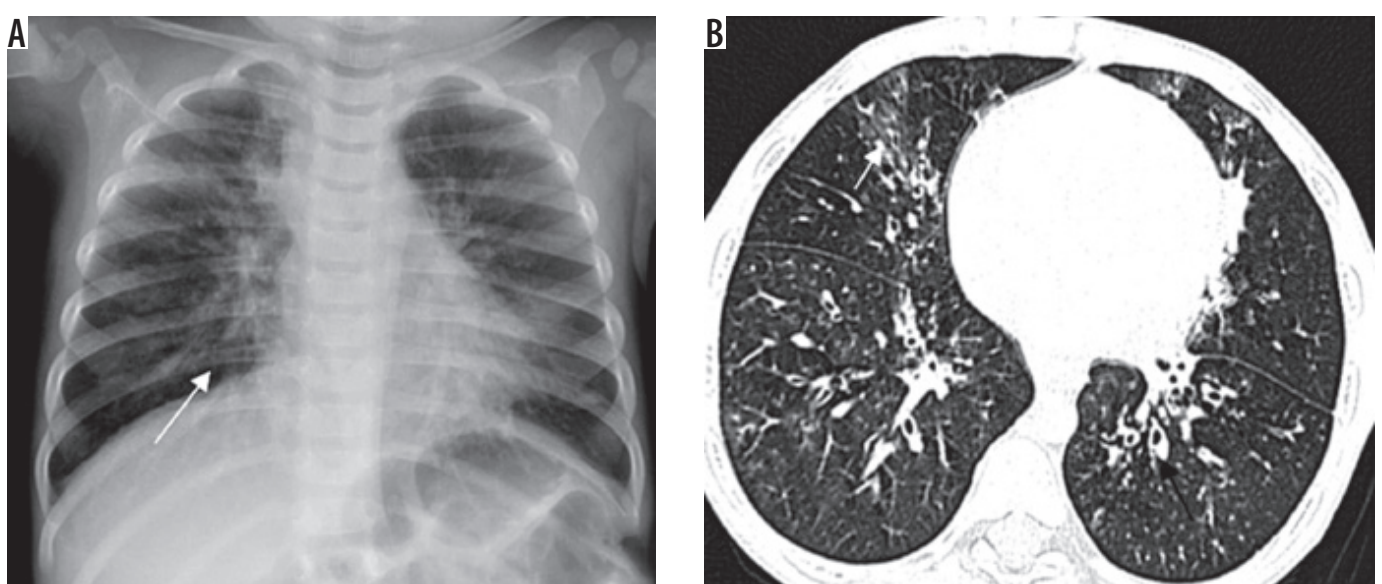

Figure 10. A) Young infant with upper respiratory infection and cough. Chest radiograph shows presence of perihilar reticulonodular opacities with peribronchial thickening (long white arrow). B) Chest computed tomography in an adult patient shows presence of patchy ground glass opacities and centrilobular nodules in right middle lobe (short white arrow), right lower lobe, left lower lobe, and lingula with associated peribronchial thickening (black arrow)
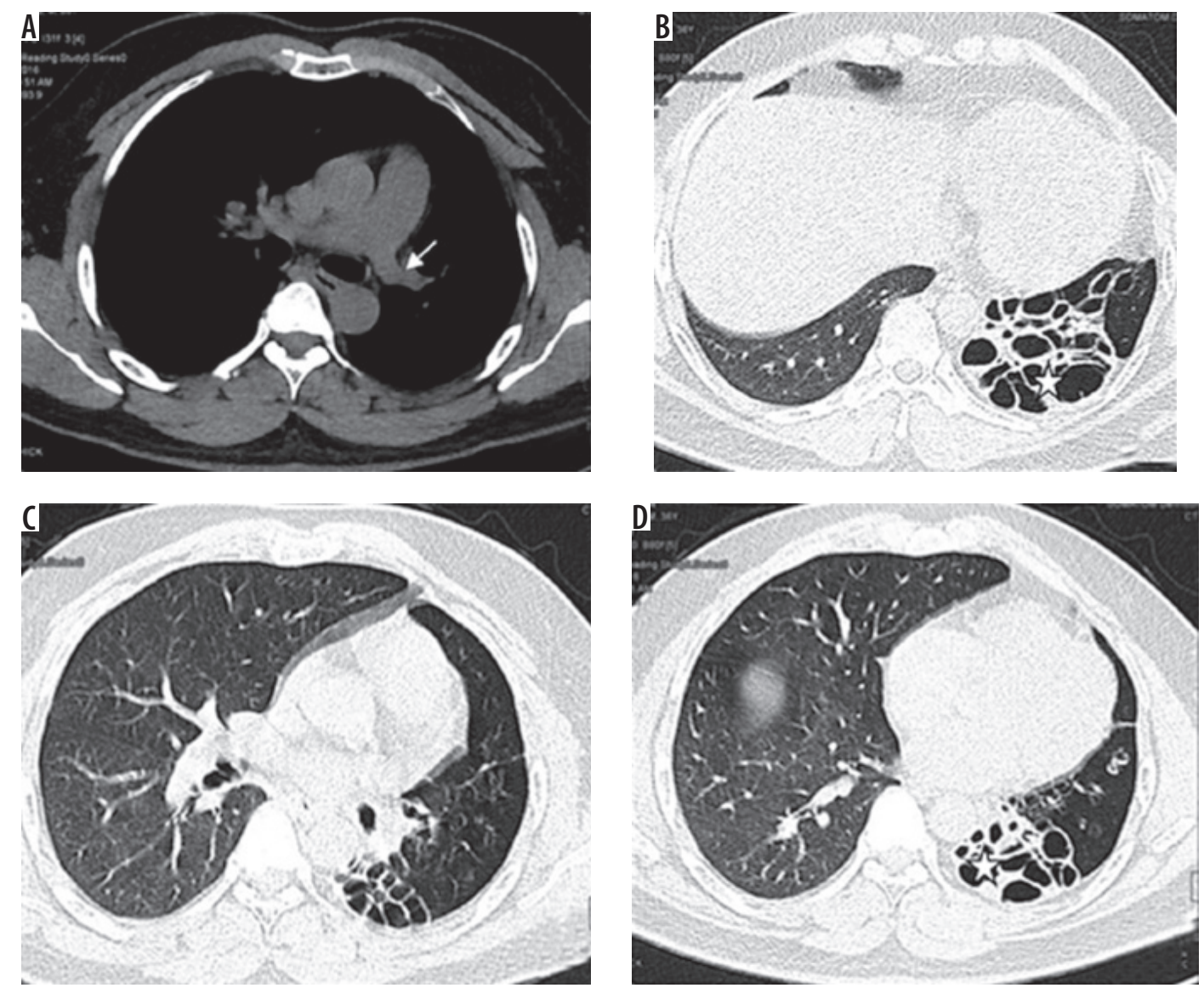

Figure 11. MacLeod's syndrome. Chest computed tomography images show presence of small-calibre left pulmonary artery (white arrow in A), associated bronchiectatic changes in left lower lobe (asterisk in B and D), and left lung hypoplasia with hyperinflated right lung

who undergo bone marrow transplant will develop CMV pneumonia [32]. CT shows consolidation, GGO, irregular reticular opacities, or centrilobular nodules (Figure 12). In AIDS patients, findings may vary, with CT showing GGO, consolidation, and discrete pulmonary nodules or masses $(1-3 \mathrm{~cm})$.

Varicella pneumonia is more common in adults than in children. Pneumonia may be concurrent with a typical skin rash. Chest radiograph shows multiple small discrete acinar nodules scattered throughout both lungs. CT also shows multiple small random nodules (Figure 13). Associated GGOs may be seen [33]. They usually resolve but rarely may persist and calcify.

\section{Conflict of interest}

The authors declare no conflict of interest. 

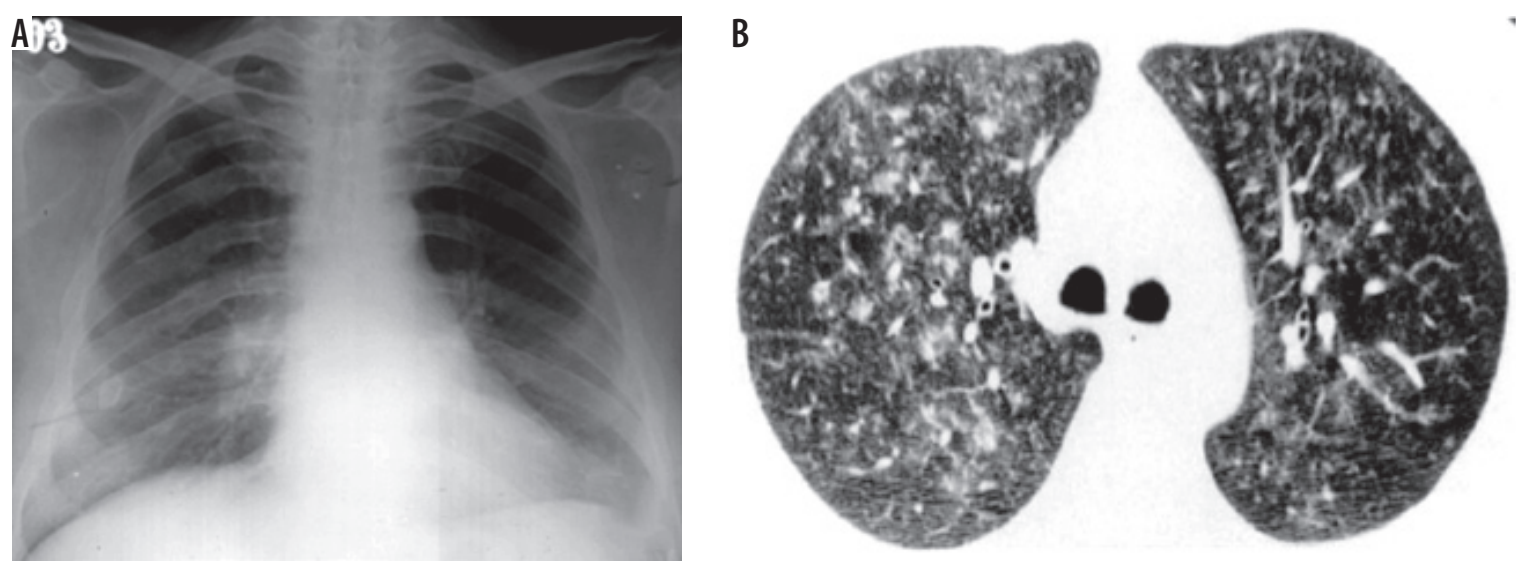

Figure 12. Cytomegalovirus pneumonia in a patient with febrile neutropenia. A) Chest radiograph shows presence of ground glass opacities in bilateral lungs. B) Chest computed tomography shows presence of diffuse ground glass opacities and ill-defined centrilobular and random nodules in bilateral lungs
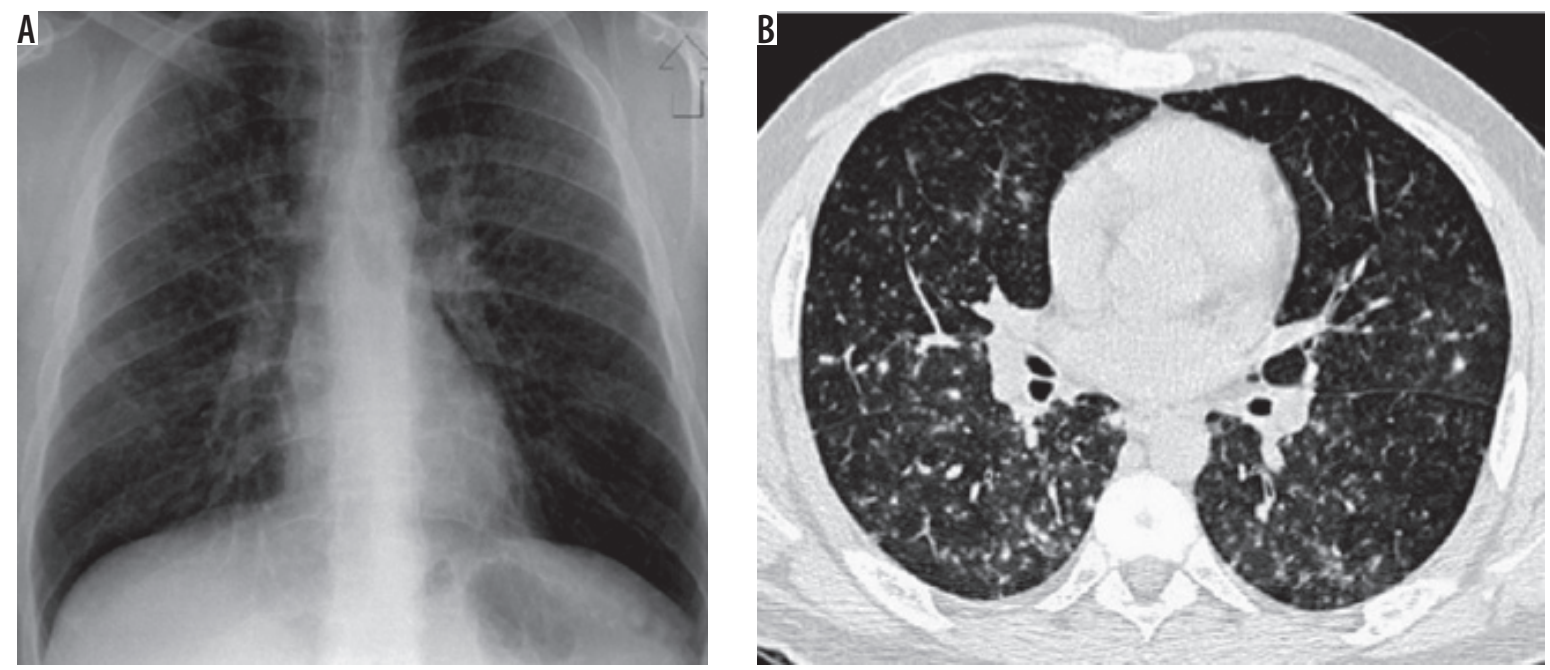

Figure 13. Patient with Varicella pneumonia. A) Chest radiograph shows presence of diffuse nodular opacities in bilateral lung fields. B) (hest computed tomography image shows presence of multiple tiny centrilobular nodules and random nodules in bilateral lungs

\section{References}

1. Padley SPG, Rubens MB. Pulmonary Infections. In: Sutton D. Textbook of Radiology and Imaging. Churchill Livingstone, Edinburgh, 2003; 131-160.

2. Sodhi KS, Khandelwal N. Magnetic Resonance Imaging of Lungs as a Radiation-Free Technique for Lung Pathologies in Immunodeficient patients. J Clin Immunol 2016; 36: 621-623.

3. Garg MK, Gupta P, Agarwal R, Sodhi KS, Khandelwal N. MRI: a new paradigm in imaging evaluation of allergic bronchopulmonary aspergillosis? Chest 2015; 147: e58-e59.

4. Reynolds JH, McDonald G, Alton H, Gordon SB. Pneumonia in the immunocompetent patient. Br J Radiol 2010; 83: 998-1009.

5. Reittner P, Ward S, Heyneman L, Johkoh T, Müller N. Pneumonia: high-resolution CT findings in 114 patients. Eur Radiol 2003; 13: 515-552.

6. American Thoracic Society. Guidelines for the management of adults with community-acquired pneumonia. Am J Respir Crit Care Med 2001; 163: 1730-1754.
7. Marik PE. Aspiration pneumonitis and aspiration pneumonia. N Engl J Med 2001; 344: 665-671.

8. Franquet T. Imaging of pneumonia: trends and algorithms. Eur Respir J 2001; 18: 196-208.

9. Okada F, Ando Y, Matsushita S, et al. Thin-section CT findings of patients with acute Streptococcus pneumoniae pneumonia with and without concurrent infection. Br J Radiol 2012; 85: e357-e364.

10. Vilar J, Domingo ML, Soto C, Cogollos J. Radiology of bacterial pneumonia. Eur J Radiol 2004; 51: 102-113.

11. Morikawa K, Okada F, Ando Y, et al. Meticillin-resistant Staphylococcus aureus and meticillin-susceptible S. aureus pneumonia: comparison of clinical and thin-section CT findings. Br J Radiol 2012; 85: e168-e175.

12. Nambu A, Saito A, Araki T, et al. Chlamydia pneumoniae: comparison with findings of Mycoplasma pneumoniae and Streptococcus pneumoniae at thin-section CT. Radiology 2006; 238: 330-338. 
13. Reittner P, Müller NL, Heyneman L, et al. Mycoplasma pneumoniae pneumonia: radiographic and high-resolution CT features in 28 patients. AJR Am J Roentgenol 2000; 174: 37-41.

14. Tanaka H. Correlation between Radiological and Pathological Findings in Patients with Mycoplasma pneumoniae Pneumonia. Front Microbiol 2016; 7: 695.

15. Sharma L, Losier A, Tolbert T, et al. Atypical Pneumonia: Updates on Legionella, Chlamydophila, and Mycoplasma Pneumonia. Clin Chest Med 2017; 38: 45-58

16. Mittal S, Singh AP, Gold M, et al. Thoracic Imaging Features of Legionnaire's Disease. Infect Dis Clin North Am 2017; 31: 43-54.

17. Okada F, Ando Y, Honda K, et al. Clinical and pulmonary thin-section CT findings in acute Klebsiella Pneumoniae pneumonia. Eur Radiol 2009; 19: 809-815.

18. Okada F, Ando Y, Honda K, et al. Acute Klebsiella pneumoniae pneumonia alone and with concurrent infection: comparison of clinical and thin-section CT findings. Br J Radiol 2010; 83: 854-860.

19. Okada F, Ono A, Ando Y, et al. Thin-section CT findings in Pseudomonas aeruginosa pulmonary infection. Br J Radiol 2012; 85: 1533-1538.

20. Beigelman-Aubry C, Godet C, Caumes E. Lung infections: the radiologist's perspective. Diagn Interv Imaging 2012; 93: 431-440.

21. Kanne JP, Yandow DR, Mohammed TL, Meyer CA. CT findings of pulmonary nocardiosis. Am J Roentgenol 2011; 197: W266-W272.

22. Daltro P, Santos EN, Gasparetto TD, et al. Pulmonary infections. Pediatr Radiol 2011; 41 Suppl 1: S69-S82.

23. Waite S, Jeudy J, White CS. Acute lung infections in normal and immunocompromised hosts. Radiol Clin North Am 2006; 44: 295-315.
24. Hollingsworth C. Thoracic disorders in the immunocompromised child. Radiol Clin North Am 2005; 43: 435-447.

25. Kloth C, Forler S, Gatidis S, et al. Comparison of chest-CT findings of Influenza virus-associated pneumonia in immunocompetent vs. immunocompromised patients. Eur J Radiol 2015; 84: 1177 1183.

26. Franquet T. Pulmonary Infections in Adults. In: Adam A, Dixon AK, Gillard JH, Schaefer-Prokop CM. Grainger \& Allison's Diagnostic Radiology. 6th Ed. Churchill Livingstone, Edinburgh 2014; 246-266.

27. Mayer JL, Lehners N, Egerer G, et al. CT-morphological characterization of respiratory syncytial virus (RSV) pneumonia in immune-compromised adults. Rofo 2014; 186: 686-692.

28. Chen HL, Chiou SS, Hsiao HP, et al. Respiratory adenoviral infections in children: a study of hospitalized cases in southern Taiwan in 2001-2002. J Trop Pediatr 2004; 50: 279-284.

29. Tan D, Fu Y, Xu J, et al. Severe adenovirus community-acquired pneumonia in immunocompetent adults: chest radiographic and CT findings. J Thorac Dis 2016; 8: 848-854.

30. Muller NL. Unilateral hyperlucent lung: MacLeod versus SwyerJames. Clin Radiol 2004; 59: 1048.

31. Chang AB, Masel JP, Masters B. Postinfectious bronchiolitis obliterans: clinical, radiological and pulmonary function sequelae. Pediatr Radiol 1998; 28: 23-29.

32. Gasparetto EL, Ono SE, Escuissato D, et al. Cytomegalovirus pneumonia after bone marrow transplantation: high resolution CT findings. Br J Radiol 2004; 77: 724-727.

33. Kim JS, Ryu CW, Lee SI, et al. High-resolution CT findings of varicella-zoster pneumonia. AJR Am J Roentgenol 1999; 172: 113-116. 